\title{
Edge-on Low Surface Brightness Galaxies in the SDSS and the Red Halo Phenomenon
}

\author{
Brady Caldwell and Nils Bergvall
}

\author{
Department of Astronomy and Space Physics, Uppsala University, Sweden \\ email: brady.caldwell@astro.uu.se, nils.bergvall@astro.uu.se
}

\begin{abstract}
Extremely red halos have been detected around high surface brightness (HSB) disk galaxies and blue compact galaxies. We analyse the halo emission of a sample of 970 stacked edge-on low surface brightness (LSB) galaxies in the SDSS (DR4) down to $\mu_{g} \sim 30 \mathrm{mag} \operatorname{arcsec}^{-2}$. These are divided by $g-r$ colour limits into a "blue" Sample A (336 galaxies), "yellow" Sample B (318 galaxies) and "red" Sample C (316 galaxies). The gri colours indicate a prominent red excess in the polar direction, strongly deviating from any normal stellar population.
\end{abstract}

\section{Results}

In Fig.1 (left) we show the colour profiles of the total sample, measured in $60^{\circ}$ wedges along the major and minor axes. We notice a dramatic reddening in the $r-i$ colours which are distinctly separated from the disk. The reddening mainly affects the galaxy along the polar direction. In Fig.1 (right) the mean colours of the faint red part of the halo in two of subsamples (data from sample A were too noisy) as well as the total sample are indicated. The figure also shows the halo colours from other studies including NGC 5907, one of the few cases where a red colour excess has been claimed for a single galaxy. Our results, although with a large scatter, seem to confirm the finding (Zibetti et al. 2004) of a red excess in halos of disk galaxies of normal types. It marginally agrees with model predictions of a bottom heavy stellar mass function (Zackrisson et al. 2006) provided the metallicity is solar or higher.

\section{References}

Zackrisson, E., et al. 2006, ApJ 650, 812.

Zibetti, S., et al. 2004, MNRAS 347, 556.
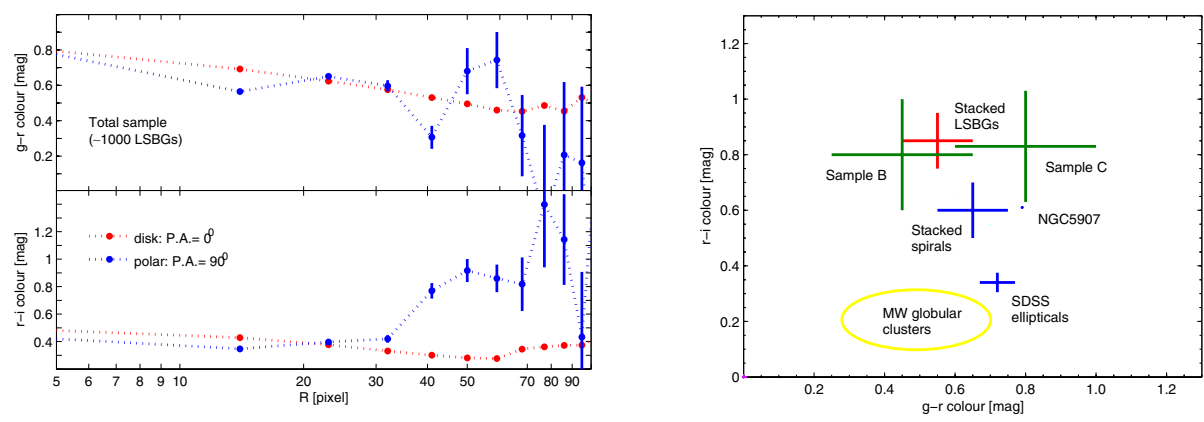

Figure 1. (left) Colour profiles: disk and polar (error bars), showing $r-i$ red excess and (right) comparison of halo colours of our data, normal ellipticals, NGC5907 and stacked spirals from SDSS (Zibetti et al. 2004). Also included are normal E galaxies and globulars 